\section{ON THE ROLE OF MICRO ANISOMETROPIA (THE “BUTTERFLY" EFFECT) IN THE OCCURRENCE OF ESOTROPIA IN CHILDREN}

\author{
Narmina .R. Hajiyeva, MD, PhD \\ National Ophthalmology Center named after the Academician \\ Zarifa Aliyeva, Baku, Azerbaijan
}

\begin{abstract}
:
Purpose: This report presents 3 cases of esotropia in children and an attempt is made to explain the cause of the development of esotropia in these children.

Methods: A comprehensive ophthalmic examination was carried out in the following order: photorefraction using the Plusoptix A09 photoscreener without cycloplegia; orthoptic examination with the alternate prism cover test; anterior segment assessment using a slit lamp; fundus examination and cycloplegic retinoscopy; and, for autorefractometry measurements, Sure-Sight.
\end{abstract}

Results: Three cases of esotropia in children are presented. In all cases, a slight microanisometropia was observed along the cylindrical component of the squinting eye.

Conclusion: Our three examples show how a slight degree of ametropia can cause a deviation of the eyeball. Therefore, case-bycase analysis can be more informative than large statistical studies. Thus, it is appropriate to evaluate each case from these new perspectives. The timely elimination of a small degree of anisometropia in the astigmatic or spherical components can lead to the restoration of the symmetrical position of the eyes.

Keywords: esotropia, infantil esotropia, strabismus, amblyopia, microanisometropia

\begin{tabular}{|l|l|}
\hline \multicolumn{2}{|c|}{ Access this article online } \\
\hline Quick Response Code: & Website: \\
\hline https://ophthalmolcases.com/index.php/ \\
\hline hat
\end{tabular}

Infantile esotropia (IE) is classically defined as an esotropia with an onset before the age of 6 months, with a large angle of strabismus, no or mild amblyopia, small to moderate hypermetropia, latent nystagmus, dissociated vertical deviation, limited abduction, and absent or reduced binocular vision in the absence of neurological disorders.1,2 IE affects between 0.25 and $0.5 \%$ of the population,3-5 depending on the degree to which children with neurological disorders are excluded. IE may have different causes, ranging from an interruption of binocular vision by eye muscle palsy to perinatal hypoxia and similar brain damage. Prematurity, low birth weight, and low Apgar scores are significant risk factors for IE.6 The ability to translate information about the disparity of the images of the two eyes into a vergence command to facilitate stereopsis is a complex cerebral function that may well falter in neurological disorders, explaining the bad outcome of strabismus surgery at a young age in such cases.7

Some cases of IE result exclusively from a motor disorder, such as eye muscle palsy or an anatomical anomaly of an eye muscle or bony orbit. In principle, such cases are more amendable to early surgery to restore or retain binocular vision.8Thus, the pathogenesis of esotropia and infantile esotropia is associated with a congenital disorder in the anatomical development of muscles and bones of the orbit, which leads to a disturbance in binocular vision. We noted that microanisometropia may be the cause of esotropia. In this report, we present the cases of 3 patients with early-onset esotropia.

\section{Case Reports}

\section{Case 1}

A 2-year-old child presented with esotropia noticed since four months of age [Figure.1.]. On the alternate prism cover test for distance, there was an esotropia of 35 prism diopters (pd) in the primary position in the right eye. The patient was otherwise healthy and neurologically normal. No family history of strabismus, amblyopia, or myopia was reported. 
A comprehensive ophthalmic examination was carried out in the following order: photorefraction using the Plusoptix A09 photoscreener without cycloplegia 8; orthoptic examination with the alternate prism cover test; , anterior segment assessment using a slit lamp; fundus examination and cycloplegic retinoscopy; and, for autorefractometry measurements, Sure-Sight.Extraocular motility was full and free in all directions of gaze.

Plusoptix(baseline): ODSph + 1, $25 \mathrm{Cyl}+\mathbf{1 . 5}$. ax $=74^{\circ} \mathrm{OS} \mathrm{Sph}+\mathbf{1 . 0}$ Cyl +1.25 ax $=115^{\circ}$

Retinoscopy: Cycloplegic refraction OD Sph + 1.0 Cyl+1.5 OS Sph +1.0 Cyl+1.0

Anterior segment findings in both eyes were normal.

In both eyes, dilated fundus examination was normal.The difference in the astigmatic component between the right eye and the left eye was 0.25-0.5 diopters. Apparently, this small difference in the asthmatic component (microanisometropia) was the triggering factor for the development of esotropia of the right eye. Wearing glasses did not work. He turned late. Therefore, he was operated on.

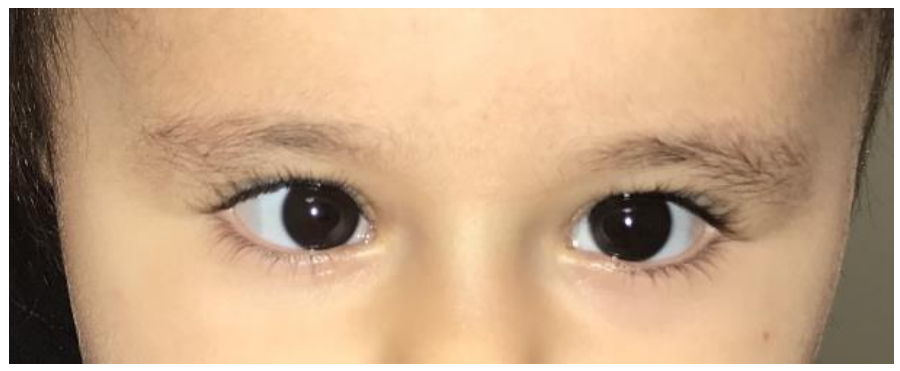

Figure.1. Esotropia of the right eye in a 2-year-old child.

\section{Case 2}

A 7-month-old child presented with esotropia noticed sincethree months of age[Fig. 2.]. On the alternate prism cover test for distance, there was an esotropia of 40 prism diopters in the primary position in the right eye. Anterior segment findingsin both eyes were normal.Dilated fundus examination was normal in both eyes.

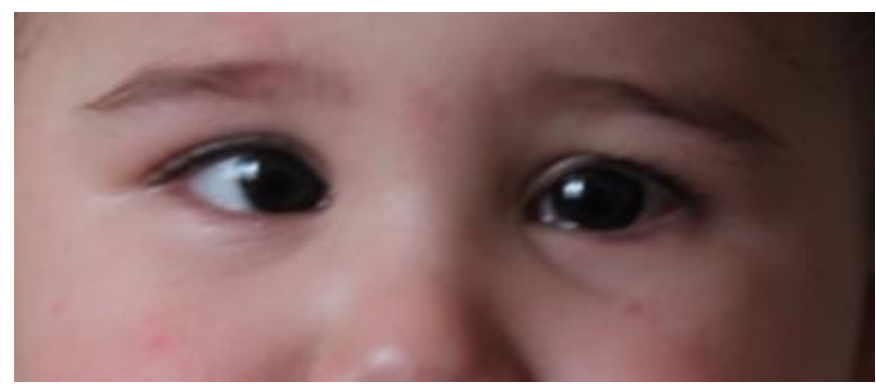

Figure 2. Child 7 months before wearing glasses

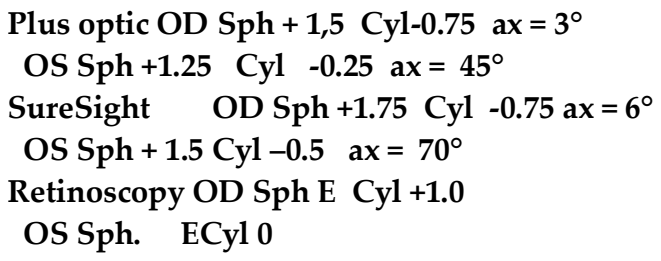

A small degree of astigmatism was confirmed by three methods of determining refraction. A slight blurring of the image on the retina was apparently the cause of the inadequate formation of binocular vision and led to deviation of the eye. The blurring of the image on the retina on one of the eyes is what interfered with the fusion of images.Spectacle correction assigned. Rp. OD Cyl +0.5 D OS Planum.Re-examination of the child after 6 months showed that the symmetrical position of the eyes had been restored [Figure. 3.].

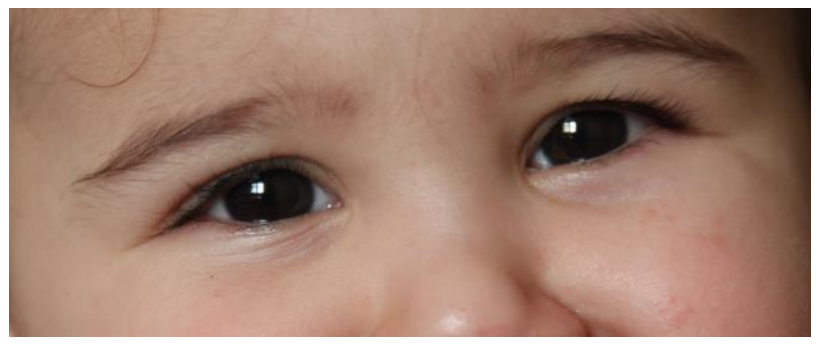

Figure .3. Child 7 months after wearing glasses.

\section{Case 3}

A 7-month-old child presented with esotropia noticed since three monthsof age. On the alternate prism cover test for distance, there was an esotropia of 35prism diopters in the primary position in the right eye. Anterior segment findingsin both eyes were normal.Dilated fundus examination was normal in both eyes

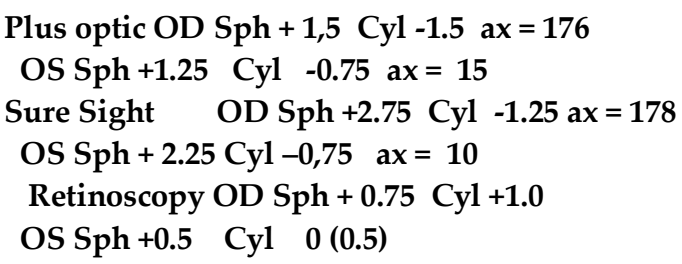

This example shows that both eyes have almost the same spherical component. The spherical component didnot affect the clarity of the image, as it is compensated by strong accommodation in the child.At the same time, microanisometropiaalong the cylindrical component can lead to some blurring of the image on the retina. This blurring is what seemedto cause the deviation of the eye Spectacle correction assigned.Rp. OD Cyl +1.25D ax= 177 OS Cyl + 0.5D ax= 12 After 6 months, the child's eyes assumed a symmetrical position. 
Figure. 5. Child 7 months before and after wearing glass

\section{Discussion}

The research devoted to refractive studies of strabismus by different authors is based on a large volume of clinical material. This material is subjected to statistical processing. However, statistical processing does not consider the minimum degree of anisotropy in the spherical and astigmatic components of refraction. Our three examples show how a slight degree of ametropia can cause a deviation of the eyeball. Therefore, case-bycase analysis can be more informative than large statistical studies. Thus, it is appropriate to evaluate each case from these new perspectives. The timely elimination of a small degree of anisotropy in the astigmatic or spherical components can lead to the restoration of the symmetrical position of the eyes. The above examples consider the most insignificant changes in the degree of astigmatism with infantile esotropia. Usually, these changes are classified as physiological, and ophthalmologists do not take them into account. Microanisometropia is one of the main factors in the occurrence of esotropia. Timely elimination of this microanisometropia can lead to the disappearance of esotropia. Similar studies have been conducted by Azerbaijani ophthalmologists.9-10

\section{Conflict of interests}

The author declares that there is no conflict of interests.

Data availability statement

The data that support the findings of this study are available from the corresponding author upon reasonable request.

\section{Funding}

None.

\section{Study association}

This study is not associated with any thesis or dissertation work 


\section{References}

1. Costenbader FD. Infantile esotropia. Trans Am Ophthalmol Soc 1961; 59:397-429.

2. Von Noorden GK. A reassessment of infantile esotropia. XLIV Edward Jackson memorial lecture. Am J Ophthalmol 1988;105(1):1-10. doi: https://doi.org/10.1016/0002- 9394(88)90113Greenberg AE, Mohney BG, Diehl NN, Burke JP. Incidence and types of childhood esotropia: a population-based study. Ophthalmology 2007;114(1):170-174. doi: 10.1016/j.ophtha.2006.05.072

3. Louwagie CR, Diehl NN, Greenberg AE, Mohney BG. Is the incidence of infantile esotropia declining?: a population-based study from Olmsted County, Minnesota, 1965 to 1994. Arch Ophthalmol 2009;127(2):200-3.

doi:

10.1001/archophthalmol.2008.568

4. Mohney BG, Erie JC, Hodge DO, Jacobsen SJ. Congenital esotropia in Olmsted County, Minnesota. Ophthalmology 1998; 105(5):846-50. doi: 10.1016/S0161-6420(98)95024-2

5. Mohney BG. Common forms of childhood strabismus in an incidence cohort. Am J Ophthalmol 2007;144(3):465-7. doi: 10.1016/j.ajo.2007.06.011

6. Charles S, Moore A. Results of early surgery for infantile esotropia in normal and neurologically impaired infants. Eye 1992;6:603-6.

7. Holman RE, Merritt JC. Infantile esotropia: results in the neurologic impaired and "normal" child at NCMH (six years). J Pediatr Ophthalmol Strabismus 1986;23(1):41-45.

https://www.ncbi.nlm.nih.gov/pubmed/3950844

8. Yan XR, Jiao WZ, Li ZW, Xu WW, Wang LH. Performance of the Plusoptix A09 Photoscreener in Detecting Amblyopia Risk Factors in Chinese Children Attending an Eye Clinic PLoS One. 2015;10(6): doi: 10.1371/journal.pone.0126052

9. Hajiyev RV, Hajiyeva NR. Anisometropia as the main factor in the development of friendly convergent strabismus in children under two years of age Oftalmologiya 2011; 5(1):64-68. (in Russian)

10. Kasimov EM, Hajiyeva NR. Early correction of esotropia in children up to 3 years. Oftalmologiya 2012;8(1):81-87.pdf (in Russian). http://oftalmologiya.az/journal/articles/2012/08/81-

How to cite this article: Hajiyeva,N. On the role of micro anisometropia (the "butterfly" effect) in the occurrence of esotropia in childre. Ophthalmology Cases \& Hypotheses. 2020;01(01):14-17. 